\title{
DIEUDONNÉ'S DETERMINANTS AND STRUCTURE OF GENERAL LINEAR GROUPS OVER DIVISION RINGS REVISITED
}

\author{
CHUN-YI LIN ${ }^{1, a}$ AND CHIA-FU YU²,b
}

\footnotetext{
${ }^{1}$ Department of Mathematics, National Taiwan University, Astronomy-Mathematics Building, No. 1, Sec. 4, Roosevelt Road, Taipei 10617, TAIWAN.

${ }^{a}$ E-mail: R09221010@ntu.edu.tw

${ }^{2}$ Institute of Mathematics, Academia Sinica and NCTS, Astronomy-Mathematics Building, No. 1, Sec. 4, Roosevelt Road, Taipei 10617, TAIWAN.

${ }^{b}$ E-mail: chiafu@math.sinica.edu.tw
}

\|\|

\begin{abstract}
In this partially expository article we revisit the construction of the Dieudonné determinant and structure of general linear groups over division rings. Our main motivation is to understand the underlying theoretic background and the proof of the simplicity of the projective special linear groups $\operatorname{PSL}_{n}(K)$ over a division ring $K$. The latter gives an important family of simple groups of Lie type. The method of proving simplicity here is based on Iwasawa's argument which proves the simplicity of $\operatorname{PSL}_{n}(F)$, where $F$ is a field. This is simpler than the proof given in E. Artin's exposition [Geometric Algebra, Interscience Publishers, 1957]. We also fix the relation on the determinants of the transposes of matrices in some literature.
\end{abstract}

\section{Introduction}

Let $n$ be a positive integer and $K$ be a division ring whose center is denoted by $Z$. Let $\mathrm{GL}_{n}(K)$ denote the group of invertible elements in the matrix algebra $\operatorname{Mat}_{n}(K)$ with entries in $K$, called the general linear group over $K$ of degree $n$. Let

$$
\Delta: \mathrm{GL}_{n}(K) \rightarrow K^{\times} /\left[K^{\times}, K^{\times}\right]
$$

Received October 29, 2020 and in revised form March 13, 2021.

AMS Subject Classification: 12E15, 16K20.

Key words and phrases: General linear groups, division rings, Dieudonné determinants. 
be the Dieudonné determinant (see [7] or Section 2 for the construction). The map $\Delta$ is a surjective group homomorphism which coincides with the usual determinant map when $K$ is commutative. The kernel of $\Delta$ is denoted by $\mathrm{SL}_{n}(K)$, called the special linear group over $K$ of degree $n$. By definition, $\mathrm{SL}_{n}(K)$ is a normal subgroup of $\mathrm{GL}_{n}(K)$ whose factor group $\mathrm{GL}_{n}(K) / \mathrm{SL}_{n}(K)$ is isomorphic to $K^{\times} /\left[K^{\times}, K^{\times}\right]$. For any group $G$, we denote by $G^{\prime}:=[G, G]$ the commutator group of $G$ and $Z(G)$ the center of $G$. Since $\mathrm{GL}_{n}(K) / \mathrm{SL}_{n}(K)$ is abelian, $\mathrm{GL}_{n}(K)^{\prime} \subset \mathrm{SL}_{n}(K)$. Let $Z_{n}(K):=Z\left(\mathrm{GL}_{n}(K)\right)$; one easily shows that $Z_{n}(K)=Z^{\times} \cdot I_{n}$ for all $n \geq 1$. We then have the inclusions:

$$
\mathrm{SL}_{n}(K)^{\prime} \subset \mathrm{GL}_{n}(K)^{\prime} \subset \mathrm{SL}_{n}(K) \subset \mathrm{GL}_{n}(K) \supset Z_{n}(K) .
$$

When $n=1$, the Dieudonné determinant $\Delta: K^{\times} \rightarrow K^{\times} /\left[K^{\times}, K^{\times}\right]$is given by the canonical projection, and one has $\left[K^{\times}, K^{\times}\right]=\mathrm{SL}_{1}(K)$. Then (1.1) becomes as follows:

$$
\left[K^{\times}, K^{\times}\right]^{\prime} \subset \mathrm{GL}_{1}(K)^{\prime}=\mathrm{SL}_{1}(K)=\left[K^{\times}, K^{\times}\right] \subset K^{\times} \supset Z^{\times} .
$$

For $n \geq 2$, we define a subgroup $E_{n}(K)$ as follows. Let $e_{i j} \in \operatorname{Mat}_{n}(K)$ be the matrix whose $(i, j)$-entry is 1 and all other entries are zero. For $1 \leq i \neq j \leq n$ and $\lambda \in K$, put $T_{i j}(\lambda)=I_{n}+\lambda e_{i j} \in \mathrm{GL}_{n}(K)$, called a transvection, and let $E_{n}(K)$ denote the subgroup of $\mathrm{GL}_{n}(K)$ generated by $T_{i j}(\lambda)$ for all $i \neq j$ and all $\lambda \in K$. One property of $\Delta$ satisfies $\Delta\left(T_{i j}(\lambda)\right)=1$ for all transvections $T_{i j}(\lambda)$. Thus, $E_{n}(K) \subset \mathrm{SL}_{n}(K)$.

The following result gives the precise relations of the subgroups $\mathrm{SL}_{n}(K)$, $E_{n}(K), \mathrm{GL}_{n}(K)^{\prime}$ and $\mathrm{SL}_{n}(K)^{\prime}$.

Theorem 1.1. Assume that $n \geq 2$. Then

(a) $E_{n}(K)=\mathrm{SL}_{n}(K)$.

(b) The proper inclusion $\mathrm{GL}_{n}(K)^{\prime} \subsetneq \mathrm{SL}_{n}(K)$ occurs exactly when $n=2$ and $|K|=2$.

(c) The proper inclusion $\mathrm{SL}_{n}(K)^{\prime} \subsetneq \mathrm{SL}_{n}(K)$ occurs exactly when $n=2$ and $|K|=2,3$. 
When $n=2$ and $K=\mathbb{F}_{3}$, one has

$$
\mathbb{F}_{3}^{\times}=Z_{2}\left(\mathbb{F}_{3}\right) \subsetneq \mathrm{SL}_{2}\left(\mathbb{F}_{3}\right)^{\prime} \cdot \mathbb{F}_{3}^{\times} \subsetneq \mathrm{GL}_{2}\left(\mathbb{F}_{3}\right)^{\prime}=\mathrm{SL}_{2}\left(\mathbb{F}_{3}\right) \subsetneq \mathrm{GL}_{2}\left(\mathbb{F}_{3}\right)
$$

whose factor groups modulo $\mathbb{F}_{3}^{\times}$are

$$
V_{4} \subset A_{4} \subset S_{4}
$$

Note that $\mathrm{SL}_{2}\left(\mathbb{F}_{3}\right)^{\prime}=\left(\mathrm{SL}_{2}\left(\mathbb{F}_{3}\right) / \mathbb{F}_{3}^{\times}\right)^{\prime} \simeq\left(A_{4}\right)^{\prime}=V_{4}$ has 4 elements and $\mathrm{SL}_{2}\left(\mathbb{F}_{3}\right)^{\prime} \cdot \mathbb{F}_{3}^{\times}$has 8 elements. Therefore, $\mathrm{SL}_{2}\left(\mathbb{F}_{3}\right)^{\prime}$ does not contain the center $\mathbb{F}_{3}^{\times}$. Notice $\mathrm{SL}_{2}\left(\mathbb{F}_{3}\right)^{\prime} \cdot \mathbb{F}_{3}^{\times}=\mathrm{SL}_{2}\left(\mathbb{F}_{3}\right)^{\prime} \times \mathbb{F}_{3}^{\times}$.

When $n=2$ and $K=\mathbb{F}_{2}$, one has

$$
\mathrm{SL}_{2}\left(\mathbb{F}_{2}\right)^{\prime}=\mathrm{GL}_{2}\left(\mathbb{F}_{2}\right)^{\prime} \subsetneq \mathrm{SL}_{2}\left(\mathbb{F}_{2}\right)=\mathrm{GL}_{2}\left(\mathbb{F}_{2}\right)
$$

$\mathrm{GL}_{2}\left(\mathbb{F}_{2}\right) \simeq S_{3}$ and $\mathrm{GL}_{2}\left(\mathbb{F}_{2}\right)^{\prime} \simeq S_{3}^{\prime}=A_{3}$

Theorem 1.2. Assume that $n \geq 2$. Let $\operatorname{PSL}_{n}(K)$ be the factor group $\mathrm{SL}_{n}(K) / Z\left(\mathrm{SL}_{n}(K)\right)$.

(a) The center $Z\left(\mathrm{SL}_{n}(K)\right)=Z^{\times} \cdot I_{n} \cap \mathrm{SL}_{n}(K)$.

(b) The group $\mathrm{PSL}_{n}(K)$ is simple if and only if $n \geq 3$, or $n=2$ and $|K|>3$.

(c) If $n \geq 3$, or $n=2$ and $|K|>3$, then every normal subgroup $G$ of $\mathrm{GL}_{n}(K)$ that is not contained in $Z_{n}(K)$ contains $\mathrm{SL}_{n}(K)$.

When $K$ is commutative, Theorems 1.1 and 1.2 are classical results; see 11] and [12, Section 6.7]. When $K$ is non-commutative, these results are also known; see below explanations. However, we could not find a single reference which includes all of them with detailed proofs.

Theorem 1.2(c) was proved in [7, Theorem 2] under a stronger assumption $n \geq 3$, or $n=2$ and $|Z|>3$. Theorem 1.2(b) was proved in [7, Theorem 3] under a stronger assumption $n \geq 3$, or $n=2$ and $|Z|>5$. Except when $n=2$ and char $K=2$, the statement $\mathrm{GL}_{n}(K)^{\prime}=E_{n}(K)$ was proved in [13, Theorem 7]; this type of result is useful; see [6]. Litoff also proved $E_{n}(R)=\mathrm{SL}_{n}(R)$ when $R$ is an Euclidean ring, but failed to discuss the question of the equality $E_{n}(K)=\mathrm{SL}_{n}(K)$ (he explained in the footnote of page 466 how the argument does not work in the non-commutative division ring case.) On the other hand, the statement $E_{n}(K)=\mathrm{SL}_{n}(K)$ is 
regarded a basic fact in this area; see [10, 2.2.2]. With this basic fact, Theorem 1.1 (b)(c) then follows immediately from [10, 2.2.3] and [13, Theorem 7]. Theorem 1.2(b) was proved in [10, Theorem 2.2.13] also under this basic fact 1 . Note that though Theorem 1.2 (c) is [3, IV, Theorem 4.9, p. 165], the proof given here, following from Theorem 1.2(b), is different from that of loc. cit. Thus, it should be clear that Theorems 1.1 and 1.2 are known from the literature. Here we provide detailed proofs of them as a convenient reference for the reader. The proof of Theorem $1.2(b)$ is close to Iwasawa's argument [11] in the case $K$ is a (commutative) field; we also refer to Jacobson's exposition [12, Chap. 6]. That is also similar as the proof given in [10].

It is worth mentioning that a proof of $E_{n}(K)=\mathrm{SL}_{n}(K)$ may have been included in the construction of the Dieudonné non-commutative determinant map. In [7] Dieudonné used the notation $C_{n}$ for two different meanings: the commutator group $\mathrm{GL}_{n}(K)^{\prime}$ of $\mathrm{GL}_{n}(K)$ and $E_{n}(K)$ in our notation. He clarified that these two subgroups are the same except when $n=2$ and $|K|=2$ (which is a consequence of Theorem 1.1 (a) and (b)); see page 32 of [7]. The group $C_{n}$ in [7, Theorem 1] is $E_{n}(K)$ but not $\mathrm{GL}_{n}(K)^{\prime}$ as stated in the Introduction of [7]. In other words, Theorem 1 of [7] reads in our notation that $\Delta$ induces an isomorphism $\Delta: \mathrm{GL}_{n}(K) / E_{n}(K) \stackrel{\sim}{\longrightarrow}$ $K^{\times} /\left[K^{\times}, K^{\times}\right]$. Therefore, $E_{n}(K)=\mathrm{SL}_{n}(K)$. In order to clarify this, we revisit the construction of the Dieudonné determinant; see Section 2.

For each matrix $A=\left(a_{i j}\right) \in \operatorname{Mat}_{n}(K)$, the transpose $A^{t}$ of $A$ is the matrix in $\operatorname{Mat}_{n}(K)$ whose $(i, j)$-entry is $a_{j i}$ for all $1 \leq i, j \leq n$. The opposite ring of $K$ is $\left(K^{\mathrm{op}}, \circ\right)$, where $K=K^{\mathrm{op}}$ as an abelian group and $a \circ b=b a$ for $a, b \in K$.

\section{Theorem 1.3.}

(1) There exist a division ring $K$ and a matrix $A \in \mathrm{GL}_{2}(K)$ such that $\Delta(A) \neq \Delta\left(A^{t}\right)$.

(2) Let $V$ be a finite right vector space over $K$, and $f \in \operatorname{End}_{K}(\mathrm{~V})$ an endomorphism of $V$ with representing matrix $A$ with respect to a fixed $K$-basis $\mathcal{B}$. Then the representing matrix of the dual endomorphism

\footnotetext{
${ }^{1}$ In the proof of 2-fold transitivity of $E_{n}(K)$ acting on the projective space in loc. cit., the argument relies on 2.2 .5 , which is simply a reformulation of 2.2 .2 via the geometric interpretation of $E_{n}(K)$ in [7, no. 4].
} 
$f^{*} \in \operatorname{End}_{\mathrm{K}}\left(\mathrm{V}^{*}\right)$ with respect to the dual basis $\mathcal{B}^{*}$ is equal to $A^{t}$ in $\operatorname{Mat}_{n}(K)$.

Moreover, if we regard $V^{*}$ as a right vector space over $K^{\mathrm{op}}$ and identify it as $\left(K^{\mathrm{op}}\right)^{n}$ using $\mathcal{B}^{*}$, then the element $f^{*} \in \operatorname{End}_{\mathrm{K}^{\circ \mathrm{p}}}\left(\mathrm{V}^{*}\right)$ corresponds to the element $A^{t} \in \operatorname{Mat}_{n}\left(K^{\mathrm{op}}\right)$.

(3) We have $(A B)^{t}=B^{t} \circ A^{t}$ for $A, B \in \operatorname{Mat}_{n}(K)$. That is, the map $\varphi: \operatorname{Mat}_{n}(K) \rightarrow \operatorname{Mat}_{n}\left(K^{\mathrm{op}}\right), A \mapsto A^{t}$ is an anti-isomorphism.

(4) We have $\Delta(A)=\Delta^{\mathrm{op}}\left(A^{t}\right)$ for every $A \in \operatorname{Mat}_{n}(K)$, where $\Delta^{\mathrm{op}}: \operatorname{Mat}_{n}\left(K^{\mathrm{op}}\right)$ $\rightarrow \overline{K^{\mathrm{op}}}$ is the Dieudonné determinant. That is, the following diagram

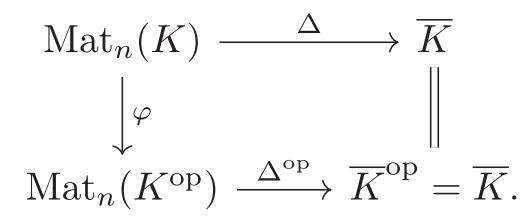

commutes.

(5) Let $D$ be a quaternion division algebra over a field $F$ with canonical involution $*$. Then for $A, B \in \operatorname{Mat}_{n}(D)$, we have $(A B)^{*}=B^{*} A^{*}$ and $\Delta(A)=\Delta\left(A^{*}\right)$, where $A^{*}$ denotes the conjugate of $A=\left(a_{i j}\right)$ whose $(i, j)$-entry is $a_{j i}^{*}$.

Theorem 1.3(1)-(4) corrects an error on the Dieudonné determinant of the transpose $A^{t}$ of a matrix $A \in \operatorname{Mat}_{n}(A)$ in [2, Theorem 1.1.4 (ii)], [1, Theorem 1.2.4 (iii)] and [4, Theorem 3.9]. We refer to [5] for further studies on skew fields and to [1] for more references discussing the Dieudonné determinant. In [18, Lemmas 8 and 9], using the Dieudonné determinant, the second author shows the connectedness of the Lie group $\mathrm{GL}_{n}(\mathbb{H})$, where $\mathbb{H}$ is the real Hamilton quaternion algebra.

This article is organized as follows. Section 2 gives the construction of the non-commutative determinant due to Diedudonné. Section 3 gives the proofs of Theorems 1.1 and 1.2. Section 4 discusses the meaning of the transpose of a matrix and its relation with the Dieudonné determinant. The proof of Theorem 1.3 is given here. 


\section{Construction of Dieudonné's Determinants and Their Properties}

In this section following [7] we shall give the construction of Dieudonn's determinants for matrices over a non-commutative division ring. Our references are [3] and [7].

Let $K$ be a division ring. For any integer $n \geq 1$, let $\operatorname{Mat}_{n}(K)$ denote the ring of square matrices of size $n$ with entries in $K$. A matrix $A \in \operatorname{Mat}_{n}(K)$ is invertible if there exists a matrix $B \in \operatorname{Mat}_{n}(K)$ such that $B A=A B=I_{n}$, where $I_{n}$ is the identity matrix. The set of all invertible matrices in $\operatorname{Mat}_{n}(K)$ forms a group, which is denoted by $\mathrm{GL}_{n}(K)$, called the general linear group of degree $n$ over $K$.

Let

$$
\bar{K}:=K /\left[K^{\times}, K^{\times}\right]=K^{\times} /\left[K^{\times}, K^{\times}\right] \cup\{0\},
$$

where $\left[K^{\times}, K^{\times}\right]$is the commutator group of $K^{\times}$. The multiplication gives a structure of monoids (not every element has the inverse) on $\bar{K}$. Our goal is to construct a function $\Delta: \operatorname{Mat}_{n}(K) \rightarrow \bar{K}$ that shares the similar properties as the usual determinant function. Namely, the following properties are satisfied:

$(\Delta 1) \Delta\left(I_{n}\right)=\overline{1}$

$(\Delta 2)$ If $A^{\prime}$ is obtained from a matrix $A \in \operatorname{Mat}_{n}(K)$ by multiplying one row on the left by $\mu$, then $\Delta\left(A^{\prime}\right)=\bar{\mu} \cdot \Delta(A)$.

$(\Delta 3)$ If $A^{\prime}$ is obtained from a matrix $A \in \operatorname{Mat}_{n}(K)$ by adding one row to another, then $\Delta\left(A^{\prime}\right)=\Delta(A)$.

$(\Delta 3)^{\prime}$ If $A^{\prime}$ is obtained from a matrix $A \in \operatorname{Mat}_{n}(K)$ with one row, say the $i$ th row $A_{i}$, replaced by $A_{i}+\mu A_{j}$ for some $\mu \in K$ and different row $A_{j}$, then $\Delta\left(A^{\prime}\right)=\Delta(A)$.

$(\Delta 4)$ If $A^{\prime}$ is obtained from a matrix $A \in \operatorname{Mat}_{n}(K)$ by exchanging two rows, then $\Delta\left(A^{\prime}\right)=\overline{-1} \cdot \Delta(A)$.

Note that the condition $(\Delta 3),(\Delta 3)^{\prime}$ or $(\Delta 4)$ is empty if $n=1$.

Lemma 2.1. Every function $\Delta: \operatorname{Mat}_{n}(K) \rightarrow \bar{K}$ which satisfies the conditions $(\Delta 1),(\Delta 2)$ and $(\Delta 3)$ also satisfies the conditions $(\Delta 3)^{\prime}$ and $(\Delta 4)$. 
Proof. Let $A \in \operatorname{Mat}_{n}(k)$, let $A_{i}$ denote the $i$ th row of $A$, and let $A^{\prime}$ be the matrix obtained from $A$ with $A_{i}$ replaced by $A_{i}+\mu A_{j}$, where $\mu \in K$. We have

$$
\begin{gathered}
\Delta(A)=\Delta\left(\left(\begin{array}{c}
\vdots \\
A_{i} \\
\vdots \\
A_{j} \\
\vdots
\end{array}\right)\right)=\bar{\mu}^{-1} \Delta\left(\left(\begin{array}{c}
\vdots \\
A_{i} \\
\vdots \\
\mu A_{j} \\
\vdots
\end{array}\right)\right) \\
\left.\left.=\left(\begin{array}{c}
A_{i}+\mu A_{j} \\
\vdots \\
\mu A_{j} \\
\vdots
\end{array}\right)\right)=\bar{\mu}^{-1} \bar{\mu} \Delta\left(\begin{array}{c}
A_{i}+\mu A_{j} \\
\vdots \\
A_{j} \\
\vdots
\end{array}\right)\right)=\Delta\left(A^{\prime}\right) .
\end{gathered}
$$

Thus, $\Delta$ satisfies the condition $(\Delta 3)^{\prime}$.

Similarly, let $A^{\prime \prime}$ be the matrix obtained from $A$ by exchanging $A_{i}$ and $A_{j}$. Then

$$
\begin{gathered}
\Delta(A)=\Delta\left(\left(\begin{array}{c}
\vdots \\
A_{i} \\
\vdots \\
A_{j} \\
\vdots
\end{array}\right)\right)=\Delta\left(\left(\begin{array}{c}
\vdots \\
A_{i}+A_{j} \\
\vdots \\
A_{j} \\
\vdots
\end{array}\right)\right)=\Delta\left(\left(\begin{array}{c}
\vdots \\
A_{i}+A_{j} \\
\vdots \\
-A_{i} \\
\vdots
\end{array}\right)\right) \\
=\Delta\left(\left(\begin{array}{c}
\vdots \\
\vdots \\
A_{j} \\
-A_{i} \\
\vdots
\end{array}\right)=\overline{-1} \cdot \Delta\left(\left(\begin{array}{c}
A_{j} \\
\vdots \\
A_{i} \\
\vdots
\end{array}\right)=\overline{-1} \cdot \Delta\left(A^{\prime \prime}\right)\right.\right.
\end{gathered}
$$

Thus, $\Delta$ satisfies the condition $(\Delta 4)$. This proves the lemma.

For $1 \leq i \neq j \leq n$ and $\lambda \in K$, let $T_{i j}(\lambda)$ be the matrix with the $(k, k)$ entry 1 for all $1 \leq k \leq n$, the $(i, j)$-entry $\lambda$, and other entries 0 . For 
any $\mu \in K$ and any integer $1 \leq i \leq n$, let $D_{i}(\mu)$ be the matrix with the $(k, k)$ entry 1 for all $k \neq i$, the $(i, i)$ entry $\mu$, and other entries 0 , that is, $D_{i}(\mu)=\operatorname{diag}(1, \ldots, \mu, \ldots, 1)$ with $\mu$ at the $i$ th position. Put $D(\mu):=D_{n}(\mu)$. One has

$$
T_{i j}(\lambda) T_{i j}\left(\lambda^{\prime}\right)=T_{i j}\left(\lambda+\lambda^{\prime}\right), \quad \text { and } \quad D_{i}(\mu) D_{i}\left(\mu^{\prime}\right)=D_{i}\left(\mu \mu^{\prime}\right)
$$

for $\lambda, \lambda^{\prime}, \mu, \mu^{\prime} \in K$. Thus, $T_{i j}(\lambda)$ and $D_{i}(\mu)$ with $\mu \in K^{\times}$are invertible matrices. For $n \geq 2$, let $E_{n}(K)$ be the subgroup of $\mathrm{GL}_{n}(K)$ generated by the invertible matrices $T_{i j}(\lambda)$ for all $i \neq j$ and all $\lambda \in K$. When $n=1$, set $E_{1}(K):=\{1\}$, the trivial subgroup.

To construct such a function $\Delta$, we give the following definition first.

Definition 2.2. A matrix $A \in \operatorname{Mat}_{n}(K)$ is said to be nonsingular if there exists a matrix $B \in \operatorname{Mat}_{n}(K)$ such that $B A=I_{n}$, and singular otherwise. Note that $A$ is nonsingular if and only if the row vectors of $A$ are left linearly independent over $K$.

Lemma 2.3. Every nonsingular matrix $A$ can be written in the form $E \cdot D(\mu)$ with $E \in E_{n}(K)$ and some $\mu \neq 0$.

Proof. There is nothing to show if $n=1$ and we assume $n \geq 2$. Since $A$ is nonsingular, not all $a_{i 1}$ are zero, and we can do a sequence of row reductions such that $a_{11}=1$ and $a_{i 1}=0$ for all $i>1$. Since the rows $A_{2}, \ldots, A_{n}$ are linearly independent, similarly we can do row reductions such that $a_{22}=1$, and $a_{i 2}=0$ for all $i \neq 2$. Do this inductively and then one gets $a_{n n}=\mu$ for some $\mu \neq 0$.

Proposition 2.4. If $A \in \operatorname{Mat}_{n}(K)$ is a nonsingular matrix with $B A=I_{n}$. Then $A B=I_{n}$. That is, $A$ is an invertible matrix.

Proof. By Lemma 2.3, $A=E \cdot D(\mu)$ is a product of two invertible matrices and hence $A$ is invertible. Therefore, $B A=A B=I_{n}$.

Proposition 2.4 says that if the row vectors of $A$ are left linearly independent then the column vectors of $A$ are right linearly independent. The similar argument using column reductions shows that the converse also holds. 
Example 2.5. Let $V$ be a vector space over a field $F$ with an infinite countable basis $x_{1}, x_{2}, \ldots$ Let $R$ be the algebra of all linear transformations from $V$ to $V$. Let $T, S$ be elements in $R$ defined by

$$
\begin{aligned}
& T\left(x_{i}\right)=x_{i+1} \quad \text { for all } i \geq 1, \quad \text { and } \\
& S\left(x_{1}\right)=x_{1}, \quad S\left(x_{i}\right)=x_{i-1} \quad \text { for all } i>1 .
\end{aligned}
$$

Then $S T=1$ but $T S \neq 1$. This shows that $T$ has a left inverse but does not have a right inverse.

Now we can construct the Dieudonn determinant $\Delta_{n}: \operatorname{Mat}_{n}(K) \rightarrow \bar{K}$ by induction on $n$ :

When $n=1$, for each $A=(a)$, set $\Delta_{1}(A):=\bar{a}$. Note that the map $\Delta_{1}$ is the unique map form $K$ to $\bar{K}$ which satisfies the conditions $(\Delta 1),(\Delta 2)$ and $(\Delta 3)$.

When $A$ is singular, set $\Delta_{n}(A):=0$. Note that this agrees with the definition when $n=1$. Let $A_{1}, \ldots, A_{n}$ be the row vectors of $A$, then $A_{1}, \ldots, A_{n}$ are left linearly dependent. Therefore, if $A^{\prime}$ is the matrix obtained from $A$ as in $(\Delta 2)$ or as in $(\Delta 3)$, then its row vectors $A_{1}^{\prime}, \ldots, A_{n}^{\prime}$ are also left linearly dependent and hence $A^{\prime}$ is singular. Therefore $\Delta_{n}\left(A^{\prime}\right)=\Delta_{n}(A)=0$ and both $(\Delta 2)$ and $(\Delta 3)$ are satisfied.

When $A$ is nonsingular, the row vectors $A_{\nu}(1 \leq \nu \leq n)$ are left linearly independent. So there exist elements $\lambda_{\nu} \in K$ such that $\sum_{\nu=1}^{n} \lambda_{\nu} A_{\nu}=e_{1}$, where $\left\{e_{1}, \ldots, e_{n}\right\}$ is the standard basis for the row vector space $K^{n}$. Write $A_{i}=\left(a_{i 1}, B_{i}\right)$, where $a_{i 1} \in K$ and $B_{i} \in K^{n-1}$, then $\Sigma_{\nu=1}^{n} \lambda_{\nu} a_{\nu 1}=1$ and $\sum_{\nu=1}^{n} \lambda_{\nu} B_{\nu}=0$. Let $B$ be the $n \times(n-1)$ matrix with rows $B_{1}, \ldots, B_{n}$, and $C_{i}$ the $(n-1) \times(n-1)$ matrix obtained from $B$ by crossing out the row $B_{i}$. Define

$$
\Delta_{n}(A):=\overline{(-1)^{i+1} \lambda_{i}^{-1}} \Delta_{n-1}\left(C_{i}\right), \quad \text { if } \lambda_{i} \neq 0
$$

We have to check that this is well-defined and the conditions $(\Delta 1),(\Delta 2)$ and $(\Delta 3)$ are satisfied.

(i) Suppose $\lambda_{i} \neq 0$ and $\lambda_{j} \neq 0$ with $i \neq j$. Let $D$ be the matrix obtained from $C_{i}$ with the row $B_{j}$ replaced by $\lambda_{j} B_{j}$, and $E$ the matrix obtained 
from $C_{i}$ with the row $B_{j}$ replaced by $B_{i}$.

By the induction hypothesis, $\Delta_{n-1}\left(C_{i}\right)=\overline{\lambda_{j}^{-1}} \Delta_{n-1}(D)$, and

$$
\begin{aligned}
& \Delta_{n-1}(D)=\Delta_{n-1}\left(\left(\begin{array}{c}
B_{1} \\
\vdots \\
\lambda_{j} B_{j} \\
\vdots \\
B_{n}
\end{array}\right)\right)=\Delta_{n-1}\left(\left(\begin{array}{c}
B_{1} \\
\vdots \\
-\Sigma_{\nu \neq i} \lambda_{\nu} B_{\nu} \\
\vdots \\
B_{n}
\end{array}\right)\right) \\
& =\Delta_{n-1}\left(\left(\begin{array}{c}
B_{1} \\
\vdots \\
-\lambda_{i} B_{i} \\
\vdots \\
B_{n}
\end{array}\right)\right)=-\overline{\lambda_{i}} \Delta_{n-1}\left(\left(\begin{array}{c}
B_{1} \\
\vdots \\
B_{i} \\
\vdots \\
B_{n}
\end{array}\right)\right)=-\overline{\lambda_{i}} \Delta_{n-1}(E) \text {. }
\end{aligned}
$$

Thus, $\Delta_{n-1}\left(C_{i}\right)=-\overline{\lambda_{j}^{-1} \lambda_{i}} \Delta_{n-1}(E)$. On the other hand, by interchanging adjacent rows $|i-j|-1$ times, we obtain another relation $\Delta_{n-1}\left(C_{j}\right)=\overline{(-1)^{i-j-1}} \Delta_{n-1}(E)$. Combining these two relations we get

$$
\overline{(-1)^{i+1} \lambda_{i}^{-1}} \Delta_{n-1}\left(C_{i}\right)=\overline{(-1)^{j+1} \lambda_{j}^{-1}} \Delta_{n-1}\left(C_{j}\right),
$$

which shows that (2.1) is independent of the choice of $i$ with $\lambda_{i} \neq 0$.

(ii) Suppose $A^{\prime}$ is obtained from a nonsingular matrix $A$ with the $i$ th row $A_{i}$ replaced by $\mu A_{i}$ for some $\mu \in K$. If $\mu=0,(\Delta 2)$ holds trivially. If $\mu \neq 0$, then $\lambda_{\nu}^{\prime}=\lambda_{\nu}$ for $\nu \neq i$ and $\lambda_{i}^{\prime}=\lambda_{i} \mu^{-1}$.

(a) If $\lambda_{i} \neq 0$, then

$$
\Delta_{n}\left(A^{\prime}\right)=\overline{(-1)^{i+1} \lambda_{i}^{\prime-1}} \Delta_{n-1}\left(C_{i}^{\prime}\right)=\overline{(-1)^{i+1} \mu \lambda_{i}^{-1}} \Delta_{n-1}\left(C_{i}\right)=\bar{\mu} \Delta_{n}(A) .
$$

(b) If $\lambda_{i}=0$ and $\lambda_{j} \neq 0$ for some $i \neq j$, then

$$
\Delta_{n}\left(A^{\prime}\right)=\overline{(-1)^{j+1} \lambda_{j}^{-1}} \Delta_{n-1}\left(C_{j}^{\prime}\right)=\overline{(-1)^{j+1} \lambda_{j}^{-1} \mu} \Delta_{n-1}\left(C_{j}\right)=\bar{\mu} \Delta_{n}(A) .
$$

(iii) Suppose $A^{\prime}$ is obtained from a nonsingular matrix $A$ with the $i$ th row $A_{i}$ replaced by $A_{i}+A_{j}$. Since $\lambda_{i}\left(A_{i}+A_{j}\right)+\left(\lambda_{j}-\lambda_{i}\right) A_{j}=\lambda_{i} A_{i}+\lambda_{j} A_{j}$, one has $\lambda_{j}^{\prime}=\lambda_{j}-\lambda_{i}$ and $\lambda_{\nu}^{\prime}=\lambda_{\nu}$ for $\nu \neq j$. 
(a) If $\lambda_{\nu} \neq 0$ for some $\nu \neq i, j$, then

$$
\Delta_{n}\left(A^{\prime}\right)=\overline{(-1)^{\nu+1} \lambda_{\nu}^{\prime-1}} \Delta_{n-1}\left(C_{\nu}^{\prime}\right)=\overline{(-1)^{\nu+1} \lambda_{\nu}^{-1}} \Delta_{n-1}\left(C_{\nu}\right)=\Delta_{n}(A) .
$$

(b) If $\lambda_{i} \neq 0$, then

$$
\Delta_{n}\left(A^{\prime}\right)=\overline{(-1)^{i+1} \lambda_{i}^{\prime-1}} \Delta_{n-1}\left(C_{i}^{\prime}\right)=\overline{(-1)^{i+1} \lambda_{i}^{-1}} \Delta_{n-1}\left(C_{i}\right)=\Delta_{n}(A) .
$$

(c) If $\lambda_{j} \neq 0$ and $\lambda_{\nu}=0$ for all $\nu \neq j$, then $B_{j}=0, B_{i}^{\prime}=B_{i}+B_{j}=B_{i}$, and $C_{j}^{\prime}=C_{j}$. Together with $\lambda_{j}^{\prime}=\lambda_{j}-\lambda_{i}=\lambda_{j}$, we obtain $\Delta_{n}\left(A^{\prime}\right)=$ $\Delta_{n}(A)$.

(iv) If $A=I_{n}$, then $\lambda_{1}=1$ and $\lambda_{\nu}=0$ for $\nu \neq 1$. Then $\Delta_{n}(A)=$ $\overline{(-1)^{2} 1^{-1}} \Delta_{n-1}\left(C_{1}\right)=\overline{1}$ by the induction hypothesis.

Theorem 2.6. Let $\Delta=\Delta_{n}: \operatorname{Mat}_{n}(K) \rightarrow \bar{K}$ be the map constructed as above.

(1) The map $\Delta$ is multiplicative, that is, one has $\Delta(A B)=\Delta(A) \Delta(B)$ for all $A, B \in \operatorname{Mat}_{n}(K)$. It is the unique map from $\operatorname{Mat}_{n}(K)$ to $\bar{K}$ which satisfies the conditions $(\Delta 1),(\Delta 2)$ and $(\Delta 3)$.

(2) When $n \geq 2$, the kernel of the group homomorphism $\Delta: \mathrm{GL}_{n}(K) \rightarrow \overline{K^{\times}}$ is equal to $E_{n}(K)$.

Proof. (1) We already showed that $\Delta$ satisfies the conditions $(\Delta 1),(\Delta 2)$ and $(\Delta 3)$, and hence it satisfies $(\Delta 3)^{\prime}$ and $(\Delta 4)$ by Lemma 2.1. It follows from the condition $(\Delta 3)^{\prime}$ that $\Delta(A B)=\Delta(B)$ for every $A \in E_{n}(K)$. By Lemma 2.3, every nonsingular matrix $A$ is equal to $E \cdot D(\mu)$ for some $E \in E_{n}(K)$, and hence $\Delta(A)=\Delta(E \cdot D(\mu))=\bar{\mu}$, by $(\Delta 3)^{\prime}$ and $(\Delta 2)$. Therefore, $\Delta$ is uniquely determined by $(\Delta 1),(\Delta 2)$ and $(\Delta 3)$. It remains to show that $\Delta$ is multiplicative.

(i) If $A$ is singular, then we must show that $A B$ is also singular. Suppose not, then by Proposition 2.4 there exists $C \in \operatorname{Mat}_{n}(K)$ such that $(A B) C=$ $I_{n}=A(B C)$; this shows that $A$ is nonsingular, a contradiction.

(ii) Suppose $A$ is nonsingular. By Lemma 2.3 we can write $A=E \cdot D(\mu)$ with $E \in E_{n}(K)$. Now $\Delta(A)=\bar{\mu}$ by $(\Delta 2)$ and $(\Delta 3)^{\prime}$, and

$$
\Delta(A B)=\Delta(E D(\mu) B)=\Delta(D(\mu) B)=\bar{\mu} \Delta(B)=\Delta(A) \Delta(B) .
$$


This proves (1).

(2) It follows from the condition $(\Delta 3)^{\prime}$ that $E_{n}(K)$ is contained in the kernel. We now show the other inclusion. Let $A=E \cdot D(\mu) \in \operatorname{ker}(\Delta)$ with $E \in$ $E_{n}(K)$, then $\mu \in\left[K^{\times}, K^{\times}\right]$. Thus, it suffices to show $D(\mu) \in E_{n}(K)$. Since $\mu$ is a product of commutators, we may assume that $\mu=a b a^{-1} b^{-1}$ for some $a, b \in K^{\times}$. For $n=2$, we can do the following row operations

$$
\begin{gathered}
\left(\begin{array}{ll}
1 & 0 \\
0 & 1
\end{array}\right) \rightarrow\left(\begin{array}{cc}
1 & 0 \\
a^{-1} & 1
\end{array}\right) \rightarrow\left(\begin{array}{cc}
0 & -a \\
a^{-1} & 1
\end{array}\right) \rightarrow\left(\begin{array}{cc}
0 & -a \\
a^{-1} & b^{-1}
\end{array}\right) \rightarrow\left(\begin{array}{cc}
a b a^{-1} & 0 \\
a^{-1} & b^{-1}
\end{array}\right) \\
\rightarrow\left(\begin{array}{cc}
a b a^{-1} & 0 \\
1 & b^{-1}
\end{array}\right) \rightarrow\left(\begin{array}{cc}
0 & -\mu \\
1 & b^{-1}
\end{array}\right) \rightarrow\left(\begin{array}{cc}
0 & -\mu \\
1 & \mu
\end{array}\right) \rightarrow\left(\begin{array}{ll}
1 & 0 \\
1 & \mu
\end{array}\right) \rightarrow\left(\begin{array}{ll}
1 & 0 \\
0 & \mu
\end{array}\right)
\end{gathered}
$$

This shows that $D(\mu) \in E_{n}(K)$. For $n>2$, the same row reductions for the last two rows shows that $D(\mu) \in E_{n}(K)$. This completes the proof.

It follows from Theorem 2.6 (2) that $E_{n}(K)$ is a normal subgroup of $\mathrm{GL}_{n}(K)$. We will give an elementary proof of this fact in Lemma 3.1, which does not rely on the construction of the Dieudonné determinant. In fact, using this lemma, we shall give another independent proof of Theorem 2.6 (2); see the proof of Theorem 1.1(a).

Lemma 2.7. Let $\iota: K_{1} \rightarrow K_{2}$ be a ring homomorphism of division rings. Then the following diagram

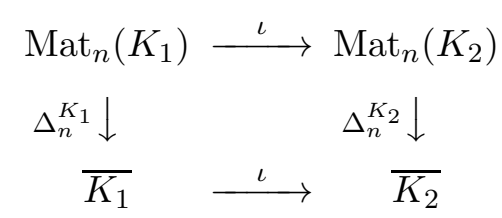

commutes.

Proof. Since $K_{1}$ is a division ring, the map $\iota$ is injective. We prove the lemma by induction on $n$. Let $A \in \operatorname{Mat}_{n}\left(K_{1}\right)$. If $A$ is singular, then $\iota(A)$ is singular and the diagram (2.2) for $A$ commutes. Clearly, when $n=1$, the diagram (2.2) commutes. Suppose $n>1$ and $A$ is nonsingular. As our construction (2.1), we have

$$
\begin{aligned}
\iota\left(\Delta_{n}(A)\right) & =\overline{(-1)^{i+1} \iota\left(\lambda_{i}\right)^{-1}} \cdot \iota\left(\Delta_{n-1}\left(C_{i}\right)\right)=\overline{(-1)^{i+1} \iota\left(\lambda_{i}\right)^{-1}} \cdot \Delta_{n-1}\left(\iota\left(C_{i}\right)\right) \\
& =\Delta_{n}(\iota(A))
\end{aligned}
$$


if $\lambda_{i} \neq 0$, where $\lambda_{\nu}$ are elements in $K_{1}$ such that $\sum_{\nu=1}^{n} \lambda_{\nu} A_{\nu}=e_{1}$. This proves the compatibility.

Lemma 2.7 shows the functorial property of the Dieudonné determinant with respect to ring homomorphisms.

\section{Structure of General Linear Groups}

\subsection{Subgroup relations}

Lemma 3.1. The subgroup $E_{n}(K)$ is normal in $\mathrm{GL}_{n}(K)$.

Proof. The proof is taken from [7] and is given for the sake of completeness. For every $g \in \mathrm{GL}_{n}(K)$, using the row reduction, there exist an element $\tau \in E_{n}(K)$ and a diagonal matrix $D(\mu)=\operatorname{diag}(1, \ldots, 1, \mu)$ with entries $1, \ldots, 1, \mu$ and $\mu \in K^{\times}$such that $g=\tau \cdot D(\mu)$. Thus, it suffices to show that every $D(\mu)$ normalizes $E_{n}(K)$. One computes

$$
\begin{aligned}
& D(\mu) T_{i j}(\lambda) D(\mu)^{-1}=T_{i j}(\lambda) \quad(i \neq n, j \neq n), \\
& D(\mu) T_{i n}(\lambda) D(\mu)^{-1}=T_{i j}\left(\lambda \mu^{-1}\right), \\
& D(\mu) T_{n j}(\lambda) D(\mu)^{-1}=T_{i j}(\mu \lambda) .
\end{aligned}
$$

Therefore, $E_{n}(K)$ is normal in $\mathrm{GL}_{n}(K)$.

Lemma 3.2. Let $K$ be a division ring. The map $K^{\times} \times K \rightarrow K,(a, c) \mapsto$ aca $-c$, is surjective if and only if $|K|>3$.

Proof. If $|K|=2,3$, then the map is $(a, c) \mapsto\left(a^{2}-1\right) c$. Since $a^{2}-1=0$ for every $a \in K^{\times}$, this is the zero map and is not surjective.

Now assume $|K|>3$. For any element $b \in K$, we claim that there is a subfield $F$ of $K$ containing $b$ such that $|F|>3$. Then we are reduced to the commutative case and the map is surjective. We first let $F$ be the subfield of $K$ generated by its center $Z$ and $b$. Then $|F|=2,3$ occurs only when $Z=\mathbb{F}_{2}$ or $Z=\mathbb{F}_{3}$, and $b \in Z$. In the latter cases we replace $F$ by the subfield generated over $Z$ by an element $d \in K$ not in $Z$. Then we have $b \in F$ and $|F|>3$, and we are done.

Proof of Theorem 1.1. (a) This is proved in Theorem 2.6(2). Here we give another independent proof using Lemma 3.1. Let $g \in \mathrm{SL}_{n}(K)$. There 
exist an element $\tau \in E_{n}(K)$ and $D(\mu)$ with $\mu \in K^{\times}$such that $g=\tau \cdot D(\mu)$. Since $g \in \mathrm{SL}_{n}(K)$ and $\Delta(\tau)=1$, one has $\mu \in\left[K^{\times}, K^{\times}\right]$. Observe from the computation

$$
\left[\begin{array}{ll}
1 & \mu \\
0 & 1
\end{array}\right]\left[\begin{array}{l}
u_{1} \\
u_{2}
\end{array}\right]=\left[\begin{array}{c}
u_{1}+\mu u_{2} \\
u_{2}
\end{array}\right], \quad\left[v_{1}, v_{2}\right]\left[\begin{array}{ll}
1 & \mu \\
0 & 1
\end{array}\right]=\left[v_{1}, v_{2}+v_{1} \mu\right],
$$

where $u_{1}, u_{2}$ are row vectors and $v_{1}, v_{2}$ are column vectors, that for a row (resp. column) reduction the scalar multiplication is on the left (resp. right). For any elements $a, d \in K^{\times}$, consider the matrix $A=\left[\begin{array}{ll}a & 0 \\ 0 & d\end{array}\right]$. Do a sequence of row reductions:

$$
\left[\begin{array}{ll}
a & 0 \\
0 & d
\end{array}\right] \rightarrow\left[\begin{array}{ll}
a & 0 \\
1 & d
\end{array}\right] \rightarrow\left[\begin{array}{cc}
1 & (1-a) d \\
1 & d
\end{array}\right] \rightarrow\left[\begin{array}{cc}
1 & (1-a) d \\
0 & a d
\end{array}\right] \rightarrow\left[\begin{array}{cc}
1 & 0 \\
0 & a d
\end{array}\right]
$$

Now we do a sequence of column reductions:

$$
\left[\begin{array}{ll}
a & 0 \\
0 & d
\end{array}\right] \rightarrow\left[\begin{array}{ll}
a & 1 \\
0 & d
\end{array}\right] \rightarrow\left[\begin{array}{cc}
1 & 1 \\
d(1-a) & d
\end{array}\right] \rightarrow\left[\begin{array}{cc}
1 & 0 \\
d(1-a) & d a
\end{array}\right] \rightarrow\left[\begin{array}{cc}
1 & 0 \\
0 & d a
\end{array}\right] .
$$

So there are elements $\tau_{1}, \tau_{2} \in E_{2}(K)$ such that $\tau_{1} A=\operatorname{diag}(1, a d)$ and $A \tau_{2}=\operatorname{diag}(1, d a)$. This implies $\operatorname{diag}\left(1, a d a^{-1} d^{-1}\right)=\tau_{1} A \tau_{2}^{-1} A^{-1} \in E_{2}(K)$ by the normality (Lemma 3.1). It follows that $D(\mu) \in E_{2}(K)$ for all $\mu \in\left[K^{\times}, K^{\times}\right]$. This proves the case $n=2$. Now replacing $A$ and $\tau_{i}$ by $\left[\begin{array}{cc}I_{n-2} & 0 \\ 0 & A\end{array}\right]$ and $\left[\begin{array}{cc}I_{n-2} & 0 \\ 0 & \tau_{i}\end{array}\right]$, respectively, the same calculation shows that $D(\mu) \in E_{n}(K)$ for every $\mu \in\left[K^{\times}, K^{\times}\right]$. Thus, $\mathrm{SL}_{n}(K)=E_{n}(K)$.

(b) When $n=2$ and $K=\mathbb{F}_{2}, \mathrm{GL}_{2}\left(\mathbb{F}_{2}\right)=\mathrm{SL}_{2}\left(\mathbb{F}_{2}\right) \simeq S_{3}$ and $\mathrm{GL}_{2}\left(\mathbb{F}_{2}\right)^{\prime} \simeq$ $\left[S_{3}, S_{3}\right]=A_{3}$ which is properly contained in $S_{3}$. Now we prove $\mathrm{GL}_{n}(K)^{\prime}=$ $E_{n}(K)$ when $n \geq 3$ or when $n=2$ and $K \neq \mathbb{F}_{2}$ and use (a) to conclude (b). If $n \geq 3$, the statement then follows from

$$
T_{i j}(\lambda)=T_{i k}(\lambda) T_{k j}(1) T_{i k}(\lambda)^{-1} T_{k j}(1)^{-1}
$$

for $i, j, k$ all distinct. Observe this shows $E_{n}(K)^{\prime}=E_{n}(K)$ when $n \geq 3$ and by (a), $\mathrm{SL}_{n}(K)=\mathrm{SL}_{n}(K)^{\prime}$. Now let $n=2$ and $K \neq \mathbb{F}_{2}$. We compute

$$
\left[\begin{array}{ll}
a & 0 \\
0 & d
\end{array}\right]\left[\begin{array}{ll}
1 & c \\
0 & 1
\end{array}\right]\left[\begin{array}{cc}
a^{-1} & 0 \\
0 & d^{-1}
\end{array}\right]\left[\begin{array}{cc}
1 & -c \\
0 & 1
\end{array}\right]=\left[\begin{array}{cc}
1 & -c+a c d^{-1} \\
0 & 1
\end{array}\right] .
$$


For any $b \in K$, we can choose $c \in K, a, d \in K^{\times}$such that $-c+a c d^{-1}=b$. For example $d=1, a \in K-\{1,0\}$ and $c=(a-1)^{-1} b$. Thus, the subgroup $\mathrm{GL}_{2}(K)^{\prime}$ contains all transvections and hence $\mathrm{GL}_{2}(K)^{\prime}=\mathrm{SL}_{2}(K)$. This completes the proof of (b).

(c) Case $n \geq 3$. By (3.1) every transvection is contained in $\mathrm{SL}_{n}(K)^{\prime}$ and hence $E_{n}(K) \subset \mathrm{SL}_{n}(K)^{\prime}$. By (a), one has $\mathrm{SL}_{n}(K) \subset \mathrm{SL}_{n}(K)^{\prime}$ and then $\mathrm{SL}_{n}(K)=\mathrm{SL}_{n}(K)^{\prime}$.

Case $n=2$. If $|K|=2,3$, then $\mathrm{SL}_{2}(K)^{\prime} \subsetneq \mathrm{SL}_{2}(K)$. Indeed, if $K=$ $\mathbb{F}_{2}$, then $\mathrm{SL}_{2}(K) \simeq S_{3}$ and $\left[S_{3}, S_{3}\right]=A_{3}$. If $K=\mathbb{F}_{3}$, then $\operatorname{PSL}_{2}(K):=$ $\mathrm{SL}_{2}(K) /\{ \pm 1\} \simeq A_{4}$ and $\left[A_{4}, A_{4}\right]=V_{4}$ (the Klein four group).

Now assume that $|K|>3$. By (3.2),

$$
\left[\begin{array}{cc}
a & 0 \\
0 & a^{-1}
\end{array}\right]\left[\begin{array}{ll}
1 & c \\
0 & 1
\end{array}\right]\left[\begin{array}{cc}
a^{-1} & 0 \\
0 & a
\end{array}\right]\left[\begin{array}{cc}
1 & -c \\
0 & 1
\end{array}\right]=\left[\begin{array}{cc}
1 & -c+a c a \\
0 & 1
\end{array}\right] .
$$

For any element $b \in K$, there exists $(a, c) \in K^{\times} \times K$ such that $a c a-c=b$ by Lemma 3.2. Thus, $T_{12}(b) \in \mathrm{SL}_{2}(F)^{\prime}$ for all $b \in K$. Similarly, we also have $T_{21}(b) \in \mathrm{SL}_{2}(K)^{\prime}$ for all $b \in K$. This shows $E_{2}(K) \subset \mathrm{SL}_{2}(K)^{\prime}$ and hence $\mathrm{SL}_{2}(K)^{\prime}=\mathrm{SL}_{2}(K)$ by $(\mathrm{a})$.

This completes the proof of Theorem 1.1.

\subsection{Simplicity of $\mathrm{PSL}_{n}(K)$}

For $n \geq 1$, let $\operatorname{PSL}_{n}(K):=\mathrm{SL}_{n}(K) / Z\left(S L_{n}(K)\right)$, called the projective special linear group over $K$ of degree $n$.

If $n=1$, then $\mathrm{SL}_{1}(K)=\left[K^{\times}, K^{\times}\right], Z\left(S L_{1}(K)\right)=Z\left(\left[K^{\times}, K^{\times}\right]\right)$and $Z_{1}(K) \cap \mathrm{SL}_{1}(K)=Z^{\times} \cap\left[K^{\times}, K^{\times}\right]$. It is not clear whether there is an inclusion relation between $Z\left(\left[K^{\times}, K^{\times}\right]\right)$and $Z^{\times} \cap\left[K^{\times}, K^{\times}\right]$in general.

Lemma 3.3. For $n \geq 2$, we have

$$
Z\left(\mathrm{SL}_{n}(K)\right)=Z_{n}(K) \cap \mathrm{SL}_{n}(K)=\left\{z \cdot I_{n} \in Z^{\times} \mid z^{n} \in\left[K^{\times}, K^{\times}\right]\right\} .
$$

In other words, the inclusion $\mathrm{SL}_{n}(K) \subset \mathrm{GL}_{n}(K)$ induces a monomorphism $\mathrm{PSL}_{n}(K) \hookrightarrow \mathrm{PGL}_{n}(K)$. 
Proof. Let $A=\left(a_{i j}\right) \in \operatorname{Mat}_{n}(K)$ be an element which commutes with all elements in $\mathrm{SL}_{n}(K)$. For each pair $1 \leq i \neq j \leq n$ and $\lambda \in K$, the relation $A \cdot T_{i j}(\lambda)=T_{i j}(\lambda) \cdot A$ gives $a_{i j} \lambda=0$ and $a_{i i} \lambda=\lambda a_{j j}$. This implies that $a_{i i}=a_{j j} \in Z$ and $a_{i j}=0$ for each pair $1 \leq i \neq j \leq n$. Thus $A=a_{11} I_{n}$.

Definition 3.4. Let $\rho: G \rightarrow \operatorname{Aut}(S)$ be an action of a group $G$ on a set $S$.

(1) If $k$ is a positive integer then the action of $G$ on $S$ is called $k$-fold transitive if for any two ordered $k$-tuples of distinct elements $\left(x_{1}, x_{2}, \ldots, x_{k}\right)$ and $\left(y_{1}, y_{2}, \ldots, y_{k}\right)$ of elements of $S$, there exists an element $g \in G$ such that $y_{i}=g x_{i}$ for $i=1, \ldots, k$.

(2) A partition $\Sigma=\left\{S_{i}\right\}_{i \in I}$ of $S$ is a collection of disjoint non-empty subsets $S_{i}$ of $S$ such that $S=\cup_{i \in I} S_{i}$. We call a partition $\Sigma$ stable under the action of $G$ if for any $i \in I$ and $g \in G$, the subset $g S_{i}$ is again a member of $\Sigma$.

(3) The action $\rho$ is said to be primitive if all the stable partitions of $S$ are $\{S\}$ and $\{\{s\}\}_{s \in S}$. In other words, it satisfies the property that if $S_{i}$ is a member of a stable partition $\Sigma$ of $S$ and $\left|S_{i}\right|>1$, then $S_{i}$ must be $S$.

Clearly, 1-fold transitivity is the same thing as transitivity. If the action $\rho$ is 2 -fold transitive, then it is primitive; see 12 , Section 6.7 , Lemma 3, p. 378].The following lemma is due to Iwasawa [11].

Lemma 3.5. Let $\rho: G \rightarrow \operatorname{Aut}(S)$ be an action of a group $G$ on a set $S$. Then the factor group $G / \operatorname{ker} \rho$ is simple if the following conditions hold

(a) $G=G^{\prime}$, the commutator group of $G$;

(b) $G$ acts primitively on $S$;

(c) There exist an element $s \in S$ and a normal abelian subgroup $A_{s}$ of the stabilizer Stabs such that $G$ is generated by the conjugates $g A_{s} g^{-1}$ for all $g \in G$.

Proof. Let $H \triangleleft G$ be a normal subgroup of $G$ and suppose $H \supsetneq \operatorname{ker} \rho$. Consider the orbits of $H$ on $S$, then $g(H s)=H(g s)$ for any $g \in G, s \in S$ by normality. Hence $G$ stabilizes the partition of $S$ into the orbits of $H$. Since $G$ acts on $S$ primitively and $H$ is not contained in $\operatorname{ker} \rho$, there is just one $H$-orbit and hence $H$ acts transitively on $S$. 
Let $g \in G$ and let $s$ be the element in condition $(c)$. Then there exists an element $h \in H$ such that $h s=g s$ by transitivity. Thus, $h^{-1} g \in \operatorname{Stab} s$ and $G=H \operatorname{Stab} s$. Now $H A_{s}$ is normal in $G=H \operatorname{Stab} s$ and contains every $g A_{s} g^{-1}$, so $G=H A_{s}$ by condition $(c)$. By isomorphism theorem $G / H \cong$ $A_{s} /\left(H \cap A_{s}\right)$, which is abelian. Therefore, $H$ contains the commutator group $G^{\prime}$ of $G$ and hence $H=G$ by condition (a). This implies that $G / \operatorname{ker} \rho$ is simple.

Let $V=K^{n}$ be the right $K$-vector space of column vectors with the standard basis $e_{1}, \ldots, e_{n}$. The left multiplication of $\operatorname{Mat}_{n}(K)$ on $V$ identifies $\operatorname{Mat}_{n}(K)$ with $\operatorname{End}_{K}(\mathrm{~V})$. Let $\mathbb{P}(V)=\mathbb{P}^{n-1}(K)$ denote the set of onedimensional $K$-subspaces in $V$, and let $\rho$ be the natural group action of $\mathrm{GL}_{n}(K)$ on $\mathbb{P}^{n-1}(K)$. We consider its restriction to the subgroup $\mathrm{SL}_{n}(K)$ acting on $\mathbb{P}^{n-1}(K)$.

Lemma 3.6. Let $n \geq 2 \in \mathbb{N}$ and $\rho$ be the natural action of $\mathrm{SL}_{n}(K)$ on $\mathbb{P}^{n-1}(K)$.

(1) The kernel of $\rho$ is $Z\left(\mathrm{SL}_{n}(K)\right)$.

(2) The action $\rho$ is 2 -fold transitive. In particularly, $\mathrm{SL}_{n}(K)$ acts primitively on $\mathbb{P}^{n-1}(K)$.

(3) The stabilizer $\operatorname{Stab}\left(e_{1} K\right)$ contains a normal abelian subgroup $A_{e_{1}}$ whose conjugates $g A_{e_{1}} g^{-1}$ for all $g \in \mathrm{SL}_{n}(K)$ generate the group $\mathrm{SL}_{n}(K)$.

Proof. (1) Let $\eta \in \operatorname{ker} \rho$. Then for every $u \in V, \eta(u)=u a_{u}$ for some $a_{u} \in$ $K$. It follows from $\eta\left(e_{i}+e_{j}\right)=e_{i} a_{e_{i}}+e_{j} a_{e_{j}}=\left(e_{i}+e_{j}\right) a_{e_{i}+e_{j}}$ that $\eta\left(e_{i}\right)=e_{i} a$ for some $a \in K$. Put $u=e_{1} \lambda+e_{2}$. Then $\eta(u)=\left(e_{1} \lambda+e_{2}\right) a_{u}=e_{1} a \lambda+e_{2} a$ and $a_{u}=a$ and $\lambda a=\lambda a$. It follows that $a \in Z$ and $\operatorname{ker} \rho=Z^{\times} \cap \mathrm{SL}_{n}(K)$.

(2) Let $x_{1} K \neq x_{2} K$ and $y_{1} K \neq y_{2} K$ be two pairs of distinct elements in $\mathbb{P}^{n-1}$. Since $x_{1}$ and $x_{2}$ (resp. $y_{1}$ and $y_{2}$ ) are linearly independent, we extend them to a basis $x_{1}, \ldots, x_{n}$ (resp. $y_{1}, \ldots, y_{n}$ ). Then there exists an element $g \in \mathrm{SL}_{n}(K)$ such that $g x_{i}=y_{i}$ for $i=1, \ldots, n-1$ and $g x_{n}=y_{n} a$ for some $a \in K$. Therefore, $\rho$ is 2 -fold transitive. 
(3) The stabilizer Stab $\left(e_{1} K\right)$ consists of matrices of the form

$$
A=\left[\begin{array}{cccc}
a_{11} & a_{12} & \ldots & a_{1 n} \\
0 & & & \\
\vdots & & A_{n-1} & \\
0 & & &
\end{array}\right]
$$

in $\mathrm{SL}_{n}(K)$. The map sending $A$ to $\left(a_{11}, A_{n-1}\right)$ defines a group homomorphism $f: \operatorname{Stab}\left(e_{1} K\right) \rightarrow K^{\times} \times \mathrm{GL}_{n-1}(K)$ with kernel

$$
A_{e_{1}}=\left\{\left[\begin{array}{llll}
1 & a_{12} & \ldots & a_{1 n} \\
0 & & & \\
\vdots & & I_{n-1} & \\
0 & & &
\end{array}\right]\right\}
$$

So $A_{e_{1}}$ is isomorphic to the additive group $\left(K^{n-1},+\right)$ and is an abelian normal subgroup of $\operatorname{Stab}\left(e_{1} K\right)$. It remains to show that $G:=\left\langle g A_{e_{1}} g^{-1} ; g \in\right.$ $\left.\mathrm{SL}_{n}(K)\right\rangle$ is $\mathrm{SL}_{n}(K)$. First, the group $A_{e_{1}}$ is generated by $T_{1 j}(b)$ for $j=$ $2, \ldots n$ and all $b \in K$. Put $P_{i j}=I_{n}-e_{i i}-e_{j j}+e_{j i}-e_{i j}$; one has $P_{12}=$ $\left[\begin{array}{cc}0 & -1 \\ 1 & 0\end{array}\right] \in \mathrm{SL}_{2}(K)$. A simple calculation

$$
\left[\begin{array}{cc}
0 & -1 \\
1 & 0
\end{array}\right] T_{12}(b)\left[\begin{array}{cc}
0 & 1 \\
-1 & 0
\end{array}\right]=T_{21}(-b),
$$

shows that $P_{i j} T_{i j}(b) P_{i j}^{-1}=T_{j i}(-b)$. When $n=2$, the group $G$ contains $T_{12}(b)$ and $T_{21}\left(b^{\prime}\right)$ for all $b, b^{\prime} \in K$ and hence is equal to $\mathrm{SL}_{2}(K)$. For $n \geq 3$, the above calculation shows that $G$ contains $T_{j 1}(b)$ for $j=2, \ldots, n$ and for all $b \in K$. For any distinct pair $i, j \geq 2$, one calculates

$$
T_{i 1}(b) T_{1 j}(1) T_{i 1}(b)^{-1} T_{1 j}(1)^{-1}=T_{i j}(b) .
$$

It follows that $G$ contains all $T_{i j}(b)$. By Theorem 1.1(a), $G=\mathrm{SL}_{n}(K)$. This completes the proof of the lemma.

Proof of Theorem 1.2, Theorem 1.2(a) is Lemma 3.3. Theorem 1.2(b) follows immediately from Theorem 1.1 and Lemmas 3.5 and 3.6. We now prove Theorem $1.2(\mathrm{c})$. 
Put $\bar{G}:=G \bmod Z_{n}(K)=Z\left(\mathrm{GL}_{n}(K)\right)$, which is a nontrivial normal subgroup of $\mathrm{PGL}_{n}(K)$ by the assumption. Then $\bar{G} \cap \mathrm{PSL}_{n}(K)$ is a normal subgroup of $\operatorname{PSL}_{n}(K)$. Suppose $\bar{G} \cap \operatorname{PSL}_{n}(K)=\{1\}$. Since $\bar{G}$ and $\mathrm{PSL}_{n}(K)$ are two normal subgroups of $\mathrm{PGL}_{n}(K)$ with trivial intersection, $\bar{G} \cdot \operatorname{PSL}_{n}(K)=\bar{G} \times \operatorname{PSL}_{n}(K)$ and hence $\bar{G}$ commutes with $\operatorname{PSL}_{n}(K)$. By Lemma 3.3, the centralizer of $\mathrm{SL}_{n}(K)$ in $\mathrm{GL}_{n}(K)$ is $Z_{n}(K)$. This implies that $G \subset Z_{n}(K)$, a contradiction. Therefore, $\bar{G} \cap \operatorname{PSL}_{n}(K)$ is a nontrivial normal subgroup of $\mathrm{PSL}_{n}(K)$ and by Theorem 1.2(b), $\bar{G} \cap \operatorname{PSL}_{n}(K)=$ $\operatorname{PSL}_{n}(K)$. Thus, $\bar{G} \supset \operatorname{PSL}_{n}(K)$ and $G \cdot Z_{n}(K) \supset \mathrm{SL}_{n}(K)$. Note that $G \supset[G, G]=\left[G \cdot Z_{n}(K), G \cdot Z_{n}(K)\right] \supset\left[\mathrm{SL}_{n}(K), \mathrm{SL}_{n}(K)\right]=\mathrm{SL}_{n}(K)$. This proves Theorem $1.2(\mathrm{c})$ and completes the proof of Theorem 1.2 .

\section{Transposes of Matrices and Their Dieudonné Determinants}

Throughout this section, $K$ denotes a division ring as in the previous sections.

\subsection{Transposes}

\section{Definition 4.1.}

(1) For each $m \times n$-matrix $A=\left(a_{i j}\right) \in \operatorname{Mat}_{m \times n}(K)$, the transpose of $A$ is the $n \times m$-matrix, denoted by $A^{t}$, in $\operatorname{Mat}_{n \times m}(K)$ whose $(i, j)$-entry is $a_{j i}$ for all $i, j$.

(2) Let $V=K^{n}$ be the standard $K$-vector space with standard basis $e_{1}, \ldots$, $e_{n}$. $V$ has a natural left and right $K$-vector space structure by

$$
\lambda\left(a_{1}, \ldots, a_{n}\right)=\left(\lambda a_{1}, \ldots, \lambda a_{n}\right), \quad\left(a_{1}, \ldots, a_{n}\right) \lambda=\left(a_{1} \lambda, \ldots, a_{n} \lambda\right)
$$

for all $a_{i} \in K$ and $\lambda \in K$. Let $f: V \rightarrow V$ be a (either left or right) $K$-linear map. The map $f$ is uniquely determined by the vectors $f\left(e_{1}\right), \ldots, f\left(e_{n}\right)$. For each $1 \leq j \leq n$, write

$$
f\left(e_{j}\right)=\sum_{i=1}^{n} a_{i j} e_{i}=\sum_{i=1}^{n} e_{i} a_{i j}, \quad a_{i j} \in K .
$$

Then $\left(a_{i j}\right)$ is called the representing matrix of $f$ with respect to the basis $e_{1}, \ldots, e_{n}$. 
(3) Suppose $V$ is a finite right $K$-vector space with basis $\mathcal{B}=\left\{e_{1}, \ldots, e_{n}\right\}$. Let $f \in \operatorname{End}_{\mathrm{K}}(\mathrm{V})$ be a $K$-linear endomorphism on $V$. For each $1 \leq j \leq n$, write

$$
f\left(e_{j}\right)=\sum_{i=1}^{n} e_{i} a_{i j}, \quad a_{i j} \in K .
$$

Then $\left(a_{i j}\right)$ is called the representing matrix of $f$ with respect to the basis $\mathcal{B}$.

(4) Suppose $W$ is a finite left $K$-vector space with basis $\mathcal{B}=\left\{e_{1}, \ldots, e_{n}\right\}$. Let $f \in \operatorname{End}_{\mathrm{K}}(\mathrm{V})$ be a $K$-linear endomorphism on $V$. For each $1 \leq j \leq n$, write

$$
f\left(e_{j}\right)=\sum_{i=1}^{n} a_{i j} e_{i}, \quad a_{i j} \in K .
$$

Then $\left(a_{i j}\right)$ is called the representing matrix of $f$ with respect to the basis $\mathcal{B}$.

When $K$ is a field, the above definitions agree with the usual definitions in linear algebra.

\section{Lemma 4.2.}

(1) If we regard $K^{n}$ as the right column vector space, and let $A=\left(a_{i j}\right)$ be the representing matrix of a $K$-linear map $f$ with respect to the standard basis $\mathcal{B}$, then for every vector $v=\left[v_{1}, \ldots, v_{n}\right]^{t} \in K^{n}$, one has

$$
f(v)=A v
$$

by the usual matrix multiplication.

(2) If we regard $K^{n}$ as the left row vector space, and let $A=\left(a_{i j}\right)$ be the representing matrix of a $K$-linear map $f$ with respect to the standard basis $\mathcal{B}$, then for every vector $v=\left[v_{1}, \ldots, v_{n}\right] \in K^{n}$, one has

$$
f(v)=v A^{t} .
$$

(3) Let $V$ (resp. $W$ ) be a right (resp. left) vector space over $K$ with a basis $\mathcal{B}\left(\right.$ resp. $\left.\mathcal{B}^{\prime}\right)$. Let $A=\left(a_{i j}\right)$ (resp. B) be the representing matrix of a $K$ linear map $f$ (resp. $g$ ) with respect to the standard basis $\mathcal{B}\left(\right.$ resp. $\left.\mathcal{B}^{\prime}\right)$. If we identify $V$ (resp. $W$ ) with the right column (resp. left row) vector space 
via $\mathcal{B}\left(\right.$ resp. $\left.\mathcal{B}^{\prime}\right)$. Then then for every vector $v=\left[v_{1}, \ldots, v_{n}\right]^{t} \in K^{n}=V$ and $w=\left[w_{1}, \ldots, w_{n}\right] \in K^{n}=W$, one has

$$
f(v)=A v, \quad \text { and } \quad g(w)=w B^{t} .
$$

Proof. The proofs are elementary and omitted.

Remark 4.3. Suppose that $K$ is non-commutative. If $A$ is a nonsingular matrix in $\operatorname{Mat}_{n}(K)$, then after doing a sequence of row reductions we obtain $A=E \cdot D(\mu)$ for some matrix $E \in E_{n}(K)$ and $\mu \in K^{\times}$. However, unlike in the commutative case, the sequence of row reductions of $A$ does not correspond a sequence of column reductions of its transpose $A^{t}$. To be precise, the multiplication by the elementary matrix $T_{12}(\mu)$ on $A$ from the left gives the row reduction with the first row $A_{1}$ replaced by $A_{1}+\mu A_{2}$ :

$$
\left(\begin{array}{ll}
1 & \mu \\
0 & 1
\end{array}\right)\left(\begin{array}{ll}
a & b \\
c & d
\end{array}\right)=\left(\begin{array}{cc}
a+\mu c & b+\mu d \\
c & d
\end{array}\right) .
$$

However, the multiplication by $T_{21}(\mu)$ on $A^{t}$ from the right gives the column reduction with the first column $C_{1}$ replaced by $C_{1}+C_{2} \mu$ (right multiple by $\mu)$ :

$$
\left(\begin{array}{ll}
a & c \\
b & d
\end{array}\right)\left(\begin{array}{ll}
1 & 0 \\
\mu & 1
\end{array}\right)=\left(\begin{array}{ll}
a+c \mu & c \\
b+d \mu & d
\end{array}\right)
$$

Therefore, the transpose of $T_{12}(\mu) A$ is not equal to $A^{t} \cdot T_{21}(\mu)$, a column reduction of $A^{t}$, unless in the special case that $\mu$ commutes with entries of $A$. In particular, in general $(A B)^{t} \neq B^{t} A^{t}$. Below we show a correct relation for the transpose of the multiplication of matrices.

\subsection{Incompatibility of the Dieudonné determinant with trans- poses}

We give an example showing that $\Delta\left(A^{t}\right)=\Delta(A)$ is not true for all $A \in \operatorname{Mat}_{n}(K)$. This gives a counterexample of [2, Theorem 1.1.4 (ii)], [1, Theorem 1.2.4 (iii)] and [4, Theorem 3.9]. 
Consider the case $n=2$, and let $K:=\mathbb{H}$ be the Hamilton quaternion algebra over $\mathbf{R}$. Denote by $1, i, j, k$ the standard basis of $\mathbb{H}$. Recall that

$$
\Delta\left(\left(\begin{array}{ll}
a & b \\
c & d
\end{array}\right)\right)=a d-a c a^{-1} b \bmod \left[\mathbb{H}^{\times}, \mathbb{H}^{\times}\right], \quad \text { if } a \neq 0 .
$$

Let $x=a d-a c a^{-1} b$ and $y=a d-a b a^{-1} c$. Since the reduced norm map $N: \mathbb{H}^{\times} \rightarrow \mathbb{R}^{\times}$factors through the quotient map $\mathbb{H}^{\times} \rightarrow \mathbb{H}^{\times} /\left[\mathbb{H}^{\times}, \mathbb{H}^{\times}\right]$, it suffices to find $a, b, c, d \in \mathbb{H}^{\times}$such that $N(x) \neq N(y)$, because $N(x) \neq N(y)$ implies $\bar{x} \neq \bar{y}$. Now take

$$
a=j, \quad b=1+i+j, \quad c=1+j-k, \quad d=1 .
$$

Then

$$
\begin{aligned}
& x=j-j(1+j-k)(-j)(1+i+j)=-2 j, \\
& y=j-j(1+i+j)(-j)(1+j-k)=2 i+2 k,
\end{aligned}
$$

and hence $N(x)=4 \neq 8=N(y)$. Thus, if we put

$$
A:=\left(\begin{array}{cc}
j & 1+i+j \\
1+j-k & 1
\end{array}\right), \quad \text { then } \Delta(A) \neq \Delta\left(A^{t}\right) .
$$

This gives a desired counterexample.

\subsection{The meaning of transposes and multiplicative relations}

Let $V$ be a finite right vector space over $K$. Fix a basis $\mathcal{B}=\left\{e_{1}, \ldots, e_{n}\right\}$. Denote by $V^{*}=\operatorname{Hom}_{K}(V, K)$ the dual vector space. It is a left vector space with scalar multiplication given by

$$
(\mu \cdot f)(v)=\mu f(v), \quad \text { for } f \in V^{*}, v \in V \text { and } \mu \in K .
$$

Let $\mathcal{B}^{*}=\left\{e_{1}^{*}, \ldots, e_{n}^{*}\right\}$ be the dual basis of $V^{*}$. That is, we have $e_{i}^{*}\left(e_{j}\right)=$ $\delta_{i j}$ for all $i, j$. For each $K$-linear endomorphism $f \in \operatorname{End}_{K}(\mathrm{~V})$, the dual $f^{*} \in \operatorname{End}_{K}\left(\mathrm{~V}^{*}\right)$ is given as the pull-back $f^{*}\left(v^{*}\right)$ of the function $v^{*}: V \rightarrow K$, namely, $f^{*}\left(v^{*}\right)(v)=v^{*}(f(v))$ for all $v \in V$. 
We denote the opposite ring of a ring $R$ by $\left(R^{\mathrm{op}}, \circ\right)$, where $R^{\mathrm{op}}=R$ as an abelian group and $a \circ b:=b a$ for $a, b \in R$. Any left $R$-module $M$ can be also reviewed as a right $R^{\mathrm{op}}$-module and vice versa. In particular, the dual vector space $V^{*}$ can be also regarded as a right vector space over $K^{\mathrm{op}}$.

Proposition 4.4. Notation being as above, let $f \in \operatorname{End}_{\mathrm{K}}(\mathrm{V})$ be an endomorphism of $V$, and $A$ the representing matrix of $f$ with respect to the basis B. Then

(1) The representing matrix of the dual endomorphism $f^{*} \in \operatorname{End}_{\mathrm{K}}\left(\mathrm{V}^{*}\right)$ with respect to the basis $\mathcal{B}^{*}$ is equal to $A^{t}$ in $\operatorname{Mat}_{n}(K)$.

(2) If we regard $V^{*}$ as a right vector space over $K^{\mathrm{op}}$ and identify it with the standard right column vector space $\left(K^{\mathrm{op}}\right)^{n}$ using the dual basis $\mathcal{B}^{*}$. Then the element $f^{*} \in \operatorname{End}_{\mathrm{K}^{\mathrm{op}}}\left(\mathrm{V}^{*}\right)=\operatorname{Mat}_{\mathrm{n}}\left(\mathrm{K}^{\mathrm{op}}\right)$ is equal to $A^{t} \in$ $\operatorname{Mat}_{n}\left(K^{\mathrm{op}}\right)=\operatorname{Mat}_{n}(K)$.

Proof. (1) Let $\mathcal{B}=\left\{e_{1}, \ldots, e_{n}\right\}, \mathcal{B}^{*}=\left\{e_{1}^{*}, \ldots, e_{n}^{*}\right\}$, and $A=\left(a_{i j}\right)$. By definition, $f\left(e_{j}\right)=\sum_{i=1}^{n} e_{i} a_{i j}$. It suffices to show that $f^{*}\left(e_{j}^{*}\right)=\sum_{i=1}^{n} a_{j i} e_{i}^{*}$, and we just need to check this for evaluating each $e_{k} \in \mathcal{B}$ :

$$
\begin{aligned}
f^{*}\left(e_{j}^{*}\right)\left(e_{k}\right) & =e_{j}^{*}\left(f\left(e_{k}\right)\right)=e_{j}^{*}\left(\sum_{i=1}^{n} e_{i} a_{i k}\right)=\sum_{i=1}^{n} e_{j}^{*}\left(e_{i}\right) a_{i k} \\
& =a_{j k}=\sum_{i=1}^{n} a_{j i} e_{i}^{*}\left(e_{k}\right) .
\end{aligned}
$$

(2) This is just the reformulation of (1) by writing elements $\sum_{i} a_{i} e_{i}^{*}$ of $V^{*}$ as $\sum_{i} e_{i}^{*} a_{i}$ and regarding $V^{*}$ as a right $K^{\text {op}}$-vector space. Namely, we need to check $f^{*}\left(e_{j}^{*}\right)=\sum_{i=1}^{n} e_{i}^{*} a_{j i}$. The computation is the same as (1) and is omitted.

Remark 4.5. Although $\operatorname{Mat}_{n}\left(K^{\mathrm{op}}\right)=\operatorname{Mat}_{n}(K)$ are the same abelian group, it is more natural to view the transpose $A^{t}$ of a matrix $A \in \operatorname{Mat}_{n}(K)$ as an element in $\operatorname{Mat}_{n}\left(K^{\mathrm{op}}\right)$ using the meaning of $A^{t}$ by Proposition 4.4(2). Then the transpose will be compatible with the matrix multiplication. On the other hand, using this interpretation it is more natural to compare $\Delta(A)$ with $\Delta^{\mathrm{op}}\left(A^{t}\right)$ rather than with $\Delta\left(A^{t}\right)$, where $\Delta^{\mathrm{op}}: \operatorname{Mat}_{n}\left(K^{\mathrm{op}}\right) \rightarrow \overline{K^{\mathrm{op}}}$ is the Dieudonné determinant. This explains the incompatibility of the Dieudonné determinant with transposes. 
Lemma 4.6. We have $(A B)^{t}=B^{t} \circ A^{t}$ for all matrices $A, B \in \operatorname{Mat}_{n}(K)$.

Proof. Let $A$ and $B$ be the representing matrices in $\operatorname{Mat}_{n}(K)=\operatorname{End}_{K}\left(\mathrm{~K}^{\mathrm{n}}\right)$ of endomorphisms $f$ and $g$ on the right column vector space $V=K^{n}$, respectively. Then $A^{t}$ and $B^{t}$ are the representing matrices in $\operatorname{Mat}_{n}\left(K^{\mathrm{op}}\right)=$ $\operatorname{End}_{K^{\circ o p}}\left(\mathrm{~V}^{*}\right)$ of the dual endomorphisms $f^{*}$ and $g^{*}$, respectively. Then $(A B)^{t}$ $\in \operatorname{Mat}_{n}\left(K^{\mathrm{op}}\right)$ is the representing matrix of $(f g)^{*}=g^{*} f^{*}$ in $\operatorname{End}_{\mathrm{K}^{\text {op }}}\left(\mathrm{V}^{*}\right)$. Therefore, $(A B)^{t}=B^{t} \circ A^{t}$.

We also give a direct elementary proof of this lemma. Write $A=\left(a_{i j}\right)$ and $B=\left(b_{j k}\right)$ with $a_{i j}, b_{j k} \in K$. Then the $(i, k)$-entry $c_{i k}$ of $A B$ is

$$
c_{i k}=\sum_{j=1}^{n} a_{i j} b_{j k} .
$$

The $(i, k)$-entry $d_{i k}$ of the matrix $B^{t} \circ A^{t}$ is

$$
d_{i k}=\sum_{j=1}^{n} b_{j i} \circ a_{k j}=\sum_{j=1} a_{k j} b_{j i}=c_{k i} .
$$

\subsection{Relation of the Dieudonné determinant with transposes}

Denote by $\Delta^{\mathrm{op}}: \operatorname{Mat}_{n}\left(K^{\mathrm{op}}\right) \rightarrow \overline{K^{\mathrm{op}}}$ the Dieudonné determinant. Let $\varphi: \operatorname{Mat}_{n}(K) \rightarrow \operatorname{Mat}_{n}\left(K^{\mathrm{op}}\right)$ be the map $A \mapsto A^{t}$. Then one has $\varphi(A B)=$ $\varphi(B) \circ \varphi(A)$, that is, $\varphi$ is an anti-isomorphism by Lemma 4.6. Note that the identity map $i d: K \rightarrow K^{\mathrm{op}}$ induces a natural identification $i d: \bar{K} \stackrel{\sim}{\longrightarrow} \overline{K^{\mathrm{op}}}$.

Proposition 4.7. We have $\Delta(A)=\Delta^{\mathrm{op}}\left(A^{t}\right)$ for every $A \in \operatorname{Mat}_{n}(K)$. That is, the following diagram

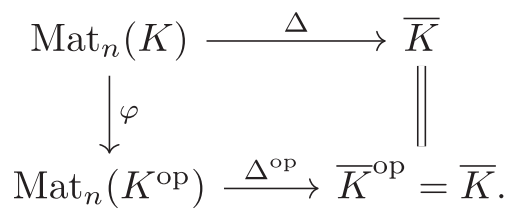

commutes.

Proof. Let $A \in \operatorname{Mat}_{n}(K)$. If $A$ is singular, then the columns of $A$ are right linearly dependent, which means that the rows of $A^{t}$ are left linearly 
dependent, i.e. $A^{t}$ is singular. Thus, in this case $\Delta(A)=0=\Delta^{\mathrm{op}}\left(A^{t}\right)$. Assume that $A$ is nonsingular. Then $A=E D(\mu)$ with $E \in E_{n}(K)$ and $\mu \in K^{\times}$. We have

$$
\Delta(A)=\bar{\mu}=\Delta^{\mathrm{op}}\left(D(\mu)^{t} \circ E^{t}\right)=\Delta^{\mathrm{op}}\left(A^{t}\right) .
$$

Note that it is not clear whether $A$ is nonsingular if and only if $A^{t}$ is nonsingular, when viewed as a matrix in $\operatorname{Mat}_{n}(K)$.

\subsection{Relation with the reduced norm maps}

Let $D$ be central simple division algebra over a field $F$. Let $\mathrm{Nr}_{n}$ : $\mathrm{GL}_{n}(D) \rightarrow F^{\times}$be the reduced norm map (restricted to $\mathrm{GL}_{n}(D)$ ) from $\operatorname{Mat}_{n}(D)$ to $F$. When $D$ is commutative, that is, $D=F$, the map $\operatorname{Nr}_{n}$ is nothing but the determinant map and is also equal to the Dieudonné determinant $\Delta_{n}$. The reduced norm $\mathrm{Nr}_{1}=\mathrm{Nr}_{D / F}: D^{\times} \rightarrow F^{\times}$induces a group homomorphism denoted again by $\mathrm{Nr}_{D / F}: D^{\times} /\left[D^{\times}, D^{\times}\right] \rightarrow F^{\times}$. Composing with the Dieudonné determinant $\Delta_{n}$, we obtain a group homomorphism:

$$
\operatorname{Nr}_{D / F} \circ \Delta_{n}: \mathrm{GL}_{n}(D) \rightarrow F^{\times} .
$$

Lemma 4.8. We have $\operatorname{Nr}_{D / F} \circ \Delta_{n}=\mathrm{Nr}_{n}$.

Proof. It is clear that the map $\mathrm{Nr}_{n}$ is characterized by the conditions that $\mathrm{Nr}_{n}(E)=1$ for $E \in E_{n}(K)$ and $\mathrm{Nr}_{n}(D(\mu))=\mathrm{Nr}_{D / F}(\mu)$ for $\mu \in D^{\times}$. It is easy to check that the group homomorphism $\mathrm{Nr}_{D / F} \circ \Delta_{n}$ satisfies these two conditions. Therefore, $\mathrm{Nr}_{D / F} \circ \Delta_{n}=\mathrm{Nr}_{n}$.

Usually it is not easy to compute the group $D^{\times} /\left[D^{\times}, D^{\times}\right]$, the target group of the Dieudonné determinant. Therefore, if the reduced norm map $\mathrm{Nr}_{D / F}$ is injective, then the Dieudonné determinant is determined by the reduced norm map $\mathrm{Nr}_{n}$. In particular, we can describe the Dieudonné determinant as a function by the reduced norm as the function from $\mathrm{GL}_{n}(D)$ to $F^{\times}$. For this direction, we have the following result due to Shianghaw Wang [17].

Theorem 4.9 (Wang). If either $F$ is a number field, or the index of $D$ over $F$, that is, $\sqrt{[D: F]}$, is square-free, then the reduced norm map $\mathrm{Nr}_{D / F}$ : $D^{\times} /\left[D^{\times}, D^{\times}\right] \rightarrow F^{\times}$is injective. 
In particular, if $D$ is a quaternion algebra, then the reduced norm map $\mathrm{Nr}_{D / F}$ is injective and one can describe the Dieudonné determinant by $\mathrm{Nr}_{n}$. In [16], Van Praag shows that if $B$ is a Hermitian matrix of degree $n$ with coefficients in $D$ then the Dieudonné determinant of $B$ has a representative that is a polynomial, independent of $n$, in the diagonal elements of $B$ and the reduced norms of elements of the subring of $D$ generated by the elements of $B$. From this he derives that the reduced norm of the Dieudonn determinant of every $n \times n$-matrix $A$ with coefficients in $D$ is a polynomial in the reduced norms of elements of the subring of $D$ generated by the entries of $A$ and their conjugates. We refer to [16] for more details.

Let $D$ be a quaternion division $F$-algebra and $*$ the canonical involution. For each $A=\left(a_{i j}\right) \in \operatorname{Mat}_{n}(D)$ denote by $A^{*}=\left(b_{i j}\right)$ the matrix with $(i, j)$ entries $b_{i j}=a_{j i}^{*}$. Then we have $(A B)^{*}=B^{*} A^{*}$.

Lemma 4.10. We have $\Delta_{n}(A)=\Delta_{n}\left(A^{*}\right)$ for all $A \in \operatorname{Mat}_{n}(D)$.

Proof. It is clear that $A$ is nonsingular if and only if $A^{*}$ is nonsingular. Therefore $\Delta_{n}(A)=\Delta_{n}\left(A^{*}\right)=0$ if $A$ is singular. If $A$ is nonsingular, then $A=E \cdot D(\mu)$ for some $E \in E_{n}(D)$ and $\mu \in D^{\times}$. Then $\Delta_{n}(A)=\bar{\mu}=$ $\Delta\left(D(\mu)^{*} E^{*}\right)=\Delta_{n}\left(A^{*}\right)$.

\section{Acknowledgments}

This article grew from lectures given by the second author in the 2019 NCTS Undergraduate Summer Research Program. He thanks the participants of the program. The authors thank Professor Ming-Chang Kang for his comments on an earlier draft of this article and Professor Cheng-Kai Liu for a helpful discussion. The second author is partially supported by the MoST grant 109-2115-M-001-002-MY3. The authors are grateful to the referee for his/her careful reading and helpful comments which eliminate several math typos and improve the exposition.

\section{References}

1. Semyon Alesker, Non-commutative linear algebra and plurisubharmonic functions of quaternionic variables, Bull. Sci. Math., 127 (2003), no. 1, 1-35. 
2. Semyon Alesker, Non-commutative determinants and quaternionic Monge-Ampere equations. Advances in analysis and geometry, 289-300, Trends Math., Birkhauser, Basel, 2004.

3. E. Artin, Geometric algebra. Interscience Publishers, Inc., New York-London, 1957.

4. J. L. Brenner, Applications of the Dieudonné determinant, Linear Algebra Appl., 1 (1968), 511-536.

5. P. M. Cohn, Skew fields. Theory of general division rings, Encyclopedia of Mathematics and its Applications, 57. Cambridge University Press, Cambridge, 1995, 500 pp.

6. Alina Carmen Cojocaru and Mihran Papikian, Drinfeld modules, Frobenius endomorphisms, and CM-liftings, Int. Math. Res. Not., IMRN 2015, no. 17, 7787-7825.

7. Jean Dieudonné, Les déterminants sur un corps non commutatif, Bull. Soc. Math. France, 71 (1943), 27-45.

8. Jean Dieudonné, On the structure of unitary groups, Trans. Amer. Math. Soc. $\mathbf{7 2}$ (1952), 367-385.

9. Jean Dieudonné, On the structure of unitary groups. II. Amer. J. Math. 75 (1953), 665-678.

10. Alexander J. Hahn and O. Timothy O'Meara, The classical groups and K-theory, Grundlehren der Mathematischen Wissenschaften, 291. Springer-Verlag, Berlin, 1989. xvi+576 pp.

11. Kenkichi Iwasawa, Uber die Einfachheit der speziellen projektiven Gruppen, Proc. Imp. Acad. Tokyo, 17 (1941), 57-59.

12. Nathan Jacobson, Basic algebra. I., Second edition, W. H. Freeman and Company, New York, 1985. xviii+499 pp.

13. O. Litoff, On the commutator subgroup of the general linear group, Proc. Amer. Math. Soc., 6 (1955), 465-470.

14. Lenny Taelman, Dieudonn determinants for skew polynomial rings, J. Algebra Appl., $\mathbf{5}$ (2006), no. 1, 89-93.

15. Tsuneo Tamagawa, On the structure of orthogonal groups, Amer. J. Math. 80 (1958), 191-197.

16. Paul Van Praag, Sur la norme réduite du déterminant de Dieudonné des matrices quaternioniennes, J. Algebra, 136 (1991), no. 2, 265-274.

17. Shianghaw Wang, On the commutator group of a simple algebra, Amer. J. Math., 72 (1950), 323-334.

18. C.-F. Yu, Notes on locally free class groups, Bull. Inst. Math. Acad. Sin. (N.S.), 12 (2017), No. 2, 125-139. 